\title{
Cromatografia em camada delgada para o diagnóstico da intoxicação por aldicarb ("chumbinho") em cães e gatos
}

\author{
[Thin-layer chromatography for aldicarb poisoning diagnosis in dogs and cats] \\ F.G. Xavier, D.A. Righi, J.C. Flório, H.S. Spinosa \\ Faculdade de Medicina Veterinária e Zootecnia - USP \\ Av. Prof. Dr. Orlando Marques de Paiva, 87 \\ 05508-900 - São Paulo, SP
}

\begin{abstract}
RESUMO
Avaliou-se a cromatografia em camada delgada (CCD) como método de diagnóstico toxicológico para os casos de intoxicação por aldicarb em cães e gatos, utilizando-se 50 amostras de conteúdo gástrico obtidas durante a necropsia e 50 amostras de alimentos utilizados como iscas para intoxicar criminalmente os animais. Todas as amostras resultaram positivas para o aldicarb, mostrando ser a CCD uma técnica qualitativa eficiente, rápida e de baixo custo, com uso potencial na toxicologia veterinária forense.
\end{abstract}

Palavras-chave: cão, gato, cromatografia em camada delgada, aldicarb, conteúdo gástrico, intoxicação

\begin{abstract}
The present study concerns about the identification of aldicarb residues using thin-layer chromatography (TLC) in 50 samples of gastric content obtained from the necropsy of dogs and cats and 50 samples of foods suspected of being used as baits. All samples resulted positive for aldicarb showing that the TLC is an efficient, fast and not expensive qualitative method for the detection of aldicarb, being useful for this purpose in the forensic veterinary toxicology.
\end{abstract}

Keywords: dog, cat, thin-layer chromatography, aldicarb, stomach content, poisoning

\section{INTRODUÇ̃̃̃O}

O aldicarb é considerado um dos praguicidas carbamatos mais tóxicos disponíveis comercialmente (Perrone et al, 1997; RagoucySengler et al., 2000). Sua aplicação e estocagem não apropriadas e distribuição ilegal têm sido apontadas como as responsáveis pelo grande número de casos de intoxicações e mortes tanto em seres humanos, como em animais (Grendom et al., 1997; Tracqui et al., 2001). Apesar de ter seu uso restrito como inseticida, acaricida e nematocida em algumas culturas agrícolas, tem sido ilegalmente utilizado como raticida doméstico no Brasil, onde é conhecido vulgarmente como "chumbinho", devido ao seu aspecto físico - pequeno grânulo cinzento, "chumbinho terrível", "raticida Japan" e "mil gatos" (Lima e Reis, 1995; Nelson et al., 2001).

Em medicina veterinária há relatos do emprego do aldicarb como agente tóxico de escolha para intoxicar intencionalmente animais domésticos, em função de sua alta toxicidade, baixo custo e fácil acesso (Frazier et al., 1999; Guitart et al., 1999; Verster et al., 2004) e também de animais silvestres, principalmente predadores, em países onde existe a caça esportiva (Motas-Guzmán et al., 2003). Em estudo recente realizado na região sudeste do Brasil sobre a casuística das intoxicações em cães e gatos (Xavier e Spinosa, 2005), o aldicarb foi o agente tóxico encontrado

Recebido em 4 de abril de 2006 
com maior freqüência, responsável por $89,0 \%$ $(113 / 117)$ das intoxicações em cães e 94,4\% (101/107), em gatos. Em todos esses casos, as intoxicações tiveram caráter intencional e os animais apresentavam indícios do agente tóxico no conteúdo gástrico ou em alimentos utilizados supostamente como iscas para este propósito criminoso.

O diagnóstico da intoxicação por aldicarb é embasado, via de regra, na avaliação clínica e na medida da atividade das colinesterases plasmática e eritrocitária. A possibilidade do emprego de análise química para a identificação do agente tóxico no material biológico de indivíduos intoxicados pode auxiliar o clínico na presteza do atendimento ao paciente e no estabelecimento de medidas de prevenção, além de ser indispensável em situações que envolvam litígios.

Dentre os métodos para análise química de agentes tóxicos, a cromatografia tem se mostrado como uma ferramenta valiosa para o isolamento e a identificação destes agentes em diversos tipos de amostras, incluindo as ambientais, embora existam poucos métodos simples para a identificação de agentes em amostras biológicas (Cardoso et al. 2001). Nesse sentido, a busca de métodos analíticos que auxiliem este propósito é importante tanto sob o ponto de vista da medicina veterinária como da saúde pública, já que a utilização indiscriminada do aldicarb em ambiente doméstico possibilita também grande risco de intoxicação em seres humanos (Lima e Reis, 1995).

Neste trabalho avaliou-se o emprego da cromatografia em camada delgada como método analítico qualitativo para o diagnóstico laboratorial rápido das intoxicações causadas pelo aldicarb em animais de companhia.

\section{MATERIAL E MÉTODOS}

Foram utilizadas 50 amostras de conteúdo gástrico obtidas durante a necropsia de cães e gatos recebidos pelo serviço de necroscopia de uma instituição de ensino de medicina veterinária, que possuíam como hipótese diagnóstica a intoxicação exógena pelo aldicarb e cujo conteúdo gástrico continha grânulos cinzentos. Após a abertura do estômago, todo o conteúdo gástrico foi colhido e mantido congelado por, no máximo seis meses, até o momento da análise. Foram também avaliadas 50 amostras de alimentos, tais como carnes, peixes, embutidos, pães etc., encaminhadas ao laboratório, que continham material sugestivo do agente tóxico aldicarb e que teriam sido utilizados como iscas para intoxicar criminalmente os animais.

O padrão técnico ${ }^{1}$ do aldicarb empregado na análise química apresentava pureza de $99,7 \%$; os reagentes empregados ${ }^{2}$ foram: diclorometano, acetona, éter de petróleo, cloreto de sódio, sulfato de sódio anidro, cloreto de platina e iodeto de potássio. Todos os procedimentos analíticos foram realizados em capela com fluxo laminar constante.

A cromatografia em camada delgada (CCD) foi feita utilizando-se como fase sólida (adsorvente) cromatoplacas tipo high performance thin-layer chromatography (HPTLC) de sílica gel 60, sem indicador fluorescente, com dimensões de $10 \mathrm{x}$ $20 \mathrm{~cm}$. A ativação da placa foi feita em estufa $\left(100^{\circ} \mathrm{C}\right)$ por, no mínimo, duas horas, antes da sua utilização.

O aldicarb foi extraído da matriz (amostra) empregando-se solução de diclorometano: acetona: éter de petróleo $(1: 1: 1)$ e cromatografado com o padrão técnico, utilizando-se como eluente o sistema solvente composto por n-hexano-acetona (4:1).

Foram consideradas amostras positivas aquelas capazes de descolorir o reagente cromogênico (iodo platina), formando pequenas manchas na mesma altura daquelas produzidas pelo padrão (mesmo Rf ou fator de retenção). A marcha analítica de cada amostra testada foi finalizada em no máximo oito horas, desde o seu descongelamento até a leitura da placa.

Para avaliar se alimentos contidos no conteúdo gástrico poderiam interferir na análise química, isto é, produzir mancha na cromatoplaca na mesma altura daquela produzida pelo aldicarb técnico, foram utilizadas sete amostras de conteúdo gástrico, sabidamente negativas,

\footnotetext{
${ }^{1}$ Aldicarb, Bayer CropScience - Charleston, EUA

${ }^{2}$ Labsynth Produtos para Laboratórios Ltda. - Diadema, Brasil
} 
obtidas de animais que vieram a óbito por outras causas. Todas elas foram submetidas em duplicata ao mesmo procedimento.

Além disso, foi determinada a menor quantidade do agente tóxico presente na amostra capaz de causar a formação de uma mancha visível na placa cromatográfica (limite de detecção). Para tanto, quantidades conhecidas do produto comercial Temik $150^{3}$ foram adicionadas em amostras de conteúdo gástrico negativas, a fim de se obter as seguintes concentrações: 10.000, $1.000,100$ e $10 \mu \mathrm{g} / \mathrm{g}$ (cerca de 1 grânulo) amostra. Uma das amostras não recebeu qualquer quantidade de aldicarb (controle negativo). Após receber o praguicida, as amostras foram homogeneizadas com bastão de vidro, mantidas em temperatura ambiente por 60 minutos e submetidas à metodologia analítica.

\section{RESULTADOS}

Todas as 50 amostras de conteúdo gástrico suspeitas de possuírem o agente tóxico, bem como as 50 amostras de alimentos suspeitos usados como iscas submetidos à CCD foram capazes de descolorir o reagente cromogênico nas regiões exatamente correspondentes àquelas descoloridas pelo padrão técnico do aldicarb, isto é, apresentaram o mesmo Rf do aldicarb.

Todas as quatro concentrações de aldicarb presente no produto comercial que foram submetidas à $\mathrm{CCD}$ produziram manchas na placa cromatográfica, cuja intensidade foi diretamente proporcional à concentração do aldicarb. Vale ressaltar que até mesmo um único grânulo foi capaz de mostrar a mancha característica na placa, indicando um limite de detecção de aproximadamente $10 \mu \mathrm{g} / \mathrm{g}$ de amostra.

\section{DISCUSSÃO}

A CCD é uma técnica qualitativa simples, barata e rápida (Wada, 1987; Baker e Poklis, 1999; Cardoso et al., 2001), descrita inicialmente em 1949 e adaptada para a toxicologia forense por Gottfried Machata no final da década de 1950 (Decker, 1987). Essa técnica está embasada na separação de substâncias através das suas

${ }^{3}$ Temik 150, Bayer CropScience - Charleston, EUA diferentes velocidades de migração em razão da afinidade relativa com solventes, fixando-se numa fase sólida.

A CCD tem sido empregada como método para a detecção do aldicarb com finalidades forenses (Spierenburg et al., 1987; Lee et al., 1999; Ameno et al., 2001; Cazenave et al., 2005), podendo servir como um método qualitativo de triagem ou mesmo como ferramenta diagnóstica nos casos de intoxicação não fatais, quando a confirmação do diagnóstico facilita a escolha do melhor regime terapêutico. Além disso, sabe-se que o estabelecimento definitivo da causa mortis nos casos periciais requer a identificação do agente tóxico em amostras biológicas obtidas do animal intoxicado.

A CCD mostrou ser uma técnica adequada para identificar o aldicarb tanto no conteúdo gástrico, como nos alimentos usados como iscas sem sofrer influência dos diferentes componentes da matriz. Ressalte-se também que até mesmo amostras mantidas congeladas por até seis meses, mostraram resultados semelhantes aos obtidos logo após o óbito do animal. Frazier et al. (1999) comentaram que a alta solubilidade dos grânulos do aldicarb no fluído gástrico, o intervalo extremamente curto entre a ingestão, o início dos sinais clínicos e a morte e o efeito estabilizante do $\mathrm{pH}$ ácido gástrico de animais monogástricos sobre o aldicarb contribuem para a preservação dessa substância na matriz biológica, permitindo sua posterior identificação química.

Outros tipos de cromatografia sugeridos para a detecção do aldicarb e seus metabólitos são a cromatografia líquida de alta resolução para amostras biológicas (Lacassie et al., 2001; Melito, 2003), água (Miles e Delfino, 1998) e vegetais (Minelli et al., 1997; Nunes et al., 1998), e a cromatografia a gás para amostras de água ou biológicas (Harper et al., 1998). No entanto, essas análises cromatográficas requerem equipamentos sofisticados, investimento em infra-estrutura e material e pessoal técnico especializado (Moraes, 2002), fatos que aumentam os custos da análise química de cada amostra e, muitas vezes, inviabilizam sua execução na rotina dos laboratórios de análises toxicológicas de países em desenvolvimento.

O limite de detecção e a seletividade são alguns dos parâmetros empregados para a validação de 
métodos analíticos (Chasin et al., 1998). O limite de detecção é a menor quantidade de agente tóxico presente na amostra capaz de produzir a formação de manchas visíveis na placa cromatográfica. No presente trabalho, todas as quatro concentrações utilizadas foram capazes de produzir resultados positivos, mostrando manchas na mesma posição daquela produzida pelo padrão técnico. A única diferença observada entre as diferentes concentrações testadas foi que a intensidade das manchas produzidas era diretamente proporcional à quantidade de aldicarb presente na amostra. $O$ limite de detecção encontrado de, aproximadamente, $10 \mu \mathrm{g} / \mathrm{g}$ de amostra se mostrou adequado, uma vez que corresponde à quantidade presente em apenas um grânulo do aldicarb encontrado no produto comercial.

A seletividade é a capacidade do método para identificar a substância de interesse na presença de outras substâncias com características semelhantes. Uma das formas de se avaliar a seletividade é mediante comparação entre a matriz isenta da substância de interesse com a matriz na qual esta substância foi adicionada (padrão); nesta última, nenhum interferente deve apresentar o tempo de retenção (Rf) da substância de interesse (Chasin et al., 1998). O método utilizado mostrou ser seletivo para a detecção do aldicarb, mesmo considerando a diversidade dos componentes presentes nos alimentos contidos no trato digestivo dos cães e gatos necropsiados e das iscas analisadas, os quais não apresentaram na CCD mancha com o mesmo Rf do aldicarb.

A CCD mostrou ser bastante adequada para o auxílio diagnóstico da intoxicação, pois todos os animais intoxicados por aldicarb apresentavam grânulos enegrecidos no conteúdo gástrico, fazendo com que a confirmação qualitativa fosse suficiente para o diagnóstico laboratorial da causa mortis.

\section{CONCLUSÃO}

O emprego da CCD para identificação do aldicarb em amostras de conteúdo gástrico ou alimentos mostrou ser uma alternativa viável de análise com finalidade diagnóstica ou forense em medicina veterinária.

\section{AGRADECIMENTOS}

Ao Conselho Nacional de Desenvolvimento Científico e Tecnológico (CNPq) pelo apoio financeiro.

\section{REFERÊNCIAS BIBLIOGRÁFICAS}

AMENO, K.; LEE, S.; IN, S. et al. Blood carbofuran concentrations in suicidal ingestion. For. Sci. Int., v.116, p.59-61, 2001.

BAKER, R.C.; POKLIS, A. Forensic toxicology: a broad overview of general principles. In: BALLANTYNE, B.; MARRS, T.; SYVERSEN, T. (Eds). General and applied toxicology. 2.ed. London: Macmillan, 1999. v.3, p.1489-1507.

CARDOSO, M.F.E.C.; COSTA, V.B.S.; DIAS, M.B. et al. Laboratório. In: ANDRADE FILHO, A.; CAMPOLINA, D. (Eds.). Toxicologia na prática clínica. Belo Horizonte: Folium, 2001. p.323-328.

CAZENAVE, S.O.S.; ITHO, S.F.; LANARO, R. et al. Aldicarb: uma possibilidade de análise com finalidade forense. Rev. Bras. Toxicol., v.18, p.105-111, 2005.

CHASIN, A.A.M.; NASCIMENTO, E.S.; RIBEIRO-NETO, L.M. et al. Validação de métodos em análises toxicológicas: uma abordagem geral. Rev. Bras. Toxicol., v.11, p.16, 1998

DECKER, W.J. Introduction and history. In: HALEY, J.J.; BERNDT, W.O. (Eds.). Handbook of toxicology. Nova York: Hemisphere Publishing Corporation, 1987. p.1-19.

FRAZIER, K.; HULLINGER, G.; HINES, M. et al. 162 cases of aldicarb intoxication in Georgia domestic animals from 1988-1998. Vet. Hum. Toxicol., v.41, p.233-235, 1999.

GRENDON, J.; FROST, F.; BAUM, L. Chronic health effects among sheep and humans surviving an aldicarb poisoning incident. Vet. Hum. Toxicol., v.36, p.218-223, 1997.

GUITART, R.; MANOSA, S.; GUERRERO, X. et al. Animal poisonings: the 10-year experience of a veterinary analytical toxicology laboratory. Vet. Hum. Toxicol., v.41, p.331-335, 1999.

HARPER, F.D.; WEISSKOPF, C.P.; COBB, G.P. Extraction of aldicarb and its metabolites 
from excreta and gastrointestinal tissue. Anal. Chem., v.70, p.3329-3332, 1998.

LACASSIE, E.; MARQUET, P.; GAULIER, J. et al. Sensitive and specific multiresidue methods for the determination of pesticides of various classes in clinical and forensic toxicology. For. Sci. Int., v.121, p.116-125, 2001.

LEE, S.; AMENO, K.; IN, S. et al. Acute fatal poisoning cases due to furathiocarb ingestion. For. Sci. Int., v.101, p.65-70, 1999.

LIMA, J.S.; REIS, C.A.G. Poisoning due to illegal use of carbamates as a rodenticide in Rio de Janeiro. Clin. Toxicol., v.33, p.687-690, 1995.

MELITO, A.L. Metodologia para identificação cromatográfica de aldicarb em sangue de cães e gatos intoxicados. 2004. 63f. Dissertação (Mestrado) - Faculdade de Medicina Veterinária e Zootecnia, Universidade de São Paulo, São Paulo.

MILES, C.J.; DELFINO, J.J. Determination of aldicarb and its derivatives in groundwater by higth-performance liquid chromatography with UV detection. J. Chromatogr., v.299, p.275-280, 1998.

MINELLI, E.V.; ANGIONI, A.; MELIS, M. et al. Determination of carbamate insecticides in apple, pears and lettuce by LC with UV detector. J. Assoc. Off. Anal. Chem., v.80, p.1315-1319, 1997.

MORAES, S.L.; REZENDE, M.O.O.; NAKAGAWA, L.E. et al. Análise de resíduos de pesticidas em tomates por cromatografia em camada delgada. Quim. Nova, v.25, p.196-202, 2002.

MOTAS-GUZMÁN, M.; MARIA-MOJICA, P.; ROMERO, D. et al. Intentional poisoning of animals in Southeastern Spain: a review of the veterinary toxicology service from Murcia, Spain. Vet. Hum. Toxicol., v.45, p.47-52, 2003.
NELSON, L.S.; PERRONE, J.; DEROOS, F. et al. Aldicarb poisoning by an illicit rodenticide imported into the United States: tres pasitos. Clin. Toxicol., v.39, p.447-452, 2001.

NUNES, G.S.; RIBEIRO, M.L.; POLESE, L. et al. Comparison of different clean-up procedures for the determination of n-methylcarbamate insecticides in vegetable matrices by high performance liquid chromatography with UV detection. J. Chromatogr. A., v.795, p.43-51, 1998.

PERRONE, J.; DEROOS, F.; MCMURRAY, W. et al. Poisonings associated with illegal use of aldicarb as a rodenticide - New York City, 19941997. J. Am. Med. Assoc., v.278, p.1487, 1997.

RAGOUCY-SENGLER, C.; TRACQUI, A.; CHAVONNET, A. et al. Aldicarb poisoning. Hum. Exp. Toxicol., v.19, p.657-662, 2000.

SPIERENBURG, T.J.; KEMMEREN-VAN DIJK, M.B.; ZOUN, P.E. High-performance liquid chromatographic analysis of aldicarb in the stomach contents of birds of prey. $J$. Chromatogr. A, v.393, p.137-9, 1987.

TRACQUI, A.; FLESCH, F.; SAUDER, P. et al. Repeated measurements organofosforados aldicarb in blood and urine in a case organofosforados nonfatal poisoning. Hum. Exp. Toxicol., v.20, p.657-660, 2001.

VERSTER, R.S.; BOTHA, C.J.; NAIDOO, V. et al. Aldicarb poisoning of dogs and cats in Gauteng during 2003. J. S. Afr. Vet. Assoc., v.75, p.177-81, 2004.

WADA, C.S. Técnicas de laboratório. 3.ed. São Paulo: Livraria Atheneu, 1987. 511p.

XAVIER F.G.; SPINOSA, H.S. Estudo das alterações post mortem macroscópicas de 214 casos de intoxicação por aldicarb ("chumbinho") em cães e gatos. Rev. Bras. Toxicol., v.18, supl., p.190, 2005. 